Article

\title{
Wheel Torque Distribution of Four-Wheel-Drive Electric Vehicles Based on Multi-Objective Optimization
}

\section{Cheng Lin ${ }^{\dagger}$ and Zhifeng $\mathrm{Xu}{ }^{\dagger} * *$}

Collaborative Innovation Center of Electric Vehicles in Beijing, Beijing Institute of Technology, Beijing 100081, China; E-Mail: lincheng@bit.edu.cn

$\dagger$ These authors contributed equally to this work.

* Author to whom correspondence should be addressed; E-Mail: xzf2012@126.com; Tel./Fax: +86-10-6891-3992.

Academic Editor: Joeri Van Mierlo

Received: 6 March 2015 / Accepted: 20 April 2015 / Published: 30 April 2015

\begin{abstract}
The wheel driving torque on four-wheel-drive electric vehicles (4WDEVs) can be modulated precisely and continuously, therefore maneuverability and energy-saving control can be carried out at the same time. In this paper, a wheel torque distribution strategy is developed based on multi-objective optimization to improve vehicle maneuverability and reduce energy consumption. In the high-layer of the presented method, sliding mode control is used to calculate the desired yaw moment due to the model inaccuracy and parameter error. In the low-layer, mathematical programming with the penalty function consisting of the yaw moment control offset, the drive system energy loss and the slip ratio constraint is used for wheel torque control allocation. The programming is solved with the combination of off-line and on-line optimization to reduce the calculation cost, and the optimization results are sent to motor controllers as torque commands. Co-simulation based on MATLAB $^{\circledR}$ and Carsim ${ }^{\circledR}$ proves that the developed strategy can both improve the vehicle maneuverability and reduce energy consumption.
\end{abstract}

Keywords: 4WDEV; wheel torque distribution; control allocation 


\section{Introduction}

As automobile emission standards are getting more stringent [1], research on and the application of electric drive technology has become a hot topic. Electric drive systems are zero emission and their output torque can be modulated precisely [2]. Based on the structure of drive system, electric vehicles can be categorized into centrally driven and distributed driven ones. The continuous and precise modulation of each wheel driving torque in the 4WDEV gives it an advantage over centrally driven vehicles when carrying out vehicle motion control and in energy savings. For example, the vehicle yaw rate can be modified through differential control of wheels on distributed driven vehicles [3], moreover, properly distributing wheel torque under different circumstances can achieve high energy efficiency which meets the energy saving needs.

The drive system structure of a four-wheel-drive electric vehicle [4] is shown in Figure 1. In this paper, a dedicated torque-vectoring controller that allows optimal wheel torque allocation is developed based on a hierarchical structure. In the high-layer, the desired driving torque and yaw moment are figured out and sent to the low-layer. In the low-layer, wheel torque is distributed for both improving the vehicle maneuverability and reducing the energy consumption.

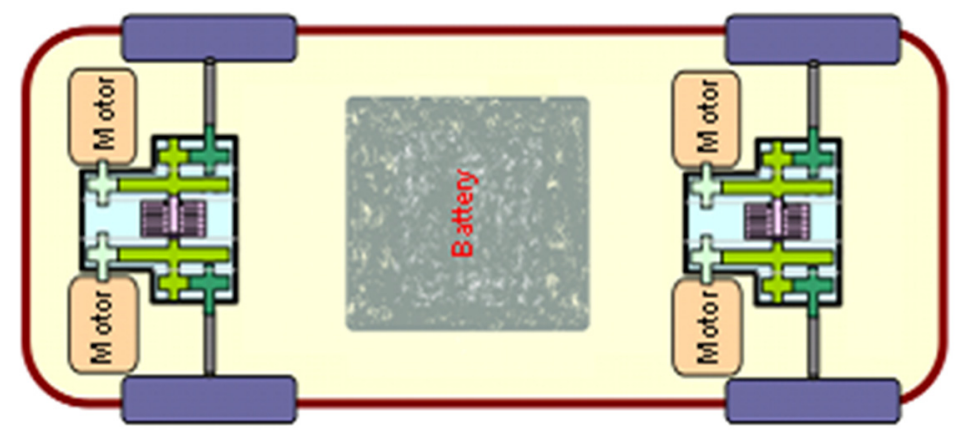

Figure 1. Drive system structure of the nominal vehicle.

As shown in the literature, many methods have been used to calculate the desired yaw moment in vehicle yaw motion control including sliding mode control [5] (SMC), fuzzy logic control [6], feed forward and feedback control [7], $H_{\infty}$ robust control [8], model predictive control [9] and adaptive control [10]. In this paper, SMC is adopted in the high-level controller due to its greater robustness to model inaccuracy and parameter error.

In terms of the wheel torque distribution layer, typical methods introduced in the literature are as follows: a torque distribution method was proposed in [11] to show the potential of optimizing the 4WDEV operational energy efficiency. The authors deduced the driving torque summation of the two wheels on the left or right side of the vehicle according to the desired yaw moment and the total driving torque, given the angle of steering wheels were approximately zero. Simulation results verified that the proposed strategy could improve the energy efficiency of the drive system, but there was no description of the influence of control strategy on vehicle maneuverability, and no slip ratio constraint was considered which may cause excessive spin of the driving wheels. In [12,13], the authors developed a wheel torque control strategy based on an optimization algorithm, and adaptive energy efficient control allocation (A-EECA) was adopted to reduce the calculation cost. The authors pointed out that the wheel torque distribution was not necessarily optimized at each time step and A-EECA can distribute wheel 
torque trending in the optimal direction at each step. As the optimization at each step was started from the last torque state, the action of torque distribution cannot follow virtual control changes in a timely way, as shown in Figure 6 of [12] and Figure 6, Figure 7 of [13]. Moreover, the influence of the controller on vehicle maneuverability was not shown clearly in the papers above. In [14], the authors pointed out that the optimal torque distribution must rely on the appropriate selection of the penalty function, and the vehicle performance provided by alternative penalty functions for the optimal wheel torque distribution of a 4WDEV were evaluated based on an off-line optimization algorithm. Results showed that using penalty functions based on the minimum tire slip criterion can achieve better vehicle performance than using functions based on energy efficiency, but the authors did not elaborate the practical algorithm for on-line application.

In this paper, the wheel torque is distributed based on multi-objective optimization. Mathematical programming is adopted with the penalty function consisting of yaw moment control offset, drive system energy loss and slip ratio constraint. The Newton-Lagrange algorithm is used to search for the optimal point on-line starting from the off-line optimization result. The on-line results are sent to the motor controllers as wheel torque commands. Co-simulation based on MATLAB ${ }^{\circledR}$ and Carsim $^{\circledR}$ verifies the proposed strategy in terms of vehicle motion control and energy saving. The rest of this paper is organized as follows: in Section 2, the proposed wheel torque control strategy is described. The high-level controller used for desired virtual control calculation is developed in Section 3. In Section 4, the wheel torque control allocation process is explained. Co-simulation results are shown in Section 5 followed by discussions. Conclusions are presented in Section 6.

\section{The Wheel Torque Control Strategy}

The work flow diagram of the proposed strategy is shown in Figure 2.In the high-layer of the strategy, the desired driving torque and yaw moment are figured out. The driver intention is interpreted first, and the desired yaw rate is obtained.

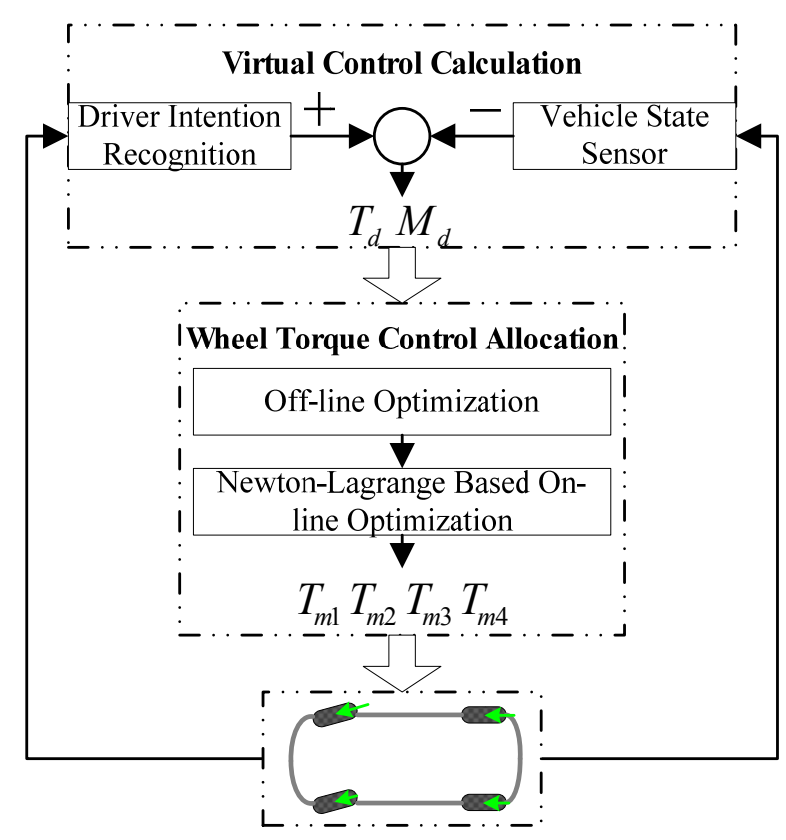

Figure 2. The wheel torque control strategy for 4WDEV. 
After that the difference between the desired yaw rate and vehicle real yaw rate observed by sensors is obtained to calculate the desired yaw moment based on SMC. In the lower-layer, the desired yaw moment and driving torque will be used in a multi-objective mathematical programming, where the penalty function consists of the yaw moment control offset, drive system energy loss and wheel slip ratio constraint. During wheel torque control allocation the maneuverability improvement and energy-saving control are both taken into consideration and the four wheels' slip ratios are balanced. As the penalty function is nonlinear and non-convex, the calculation cost of algorithms to figure out the global optimal point is too high for on-line application. In this paper, off-line optimization based on a simplified penalty function is used to preliminarily get an optimal point, and the following on-line optimization is used to search for the local optimal point starting from the off-line result. The results of the on-line optimization will be used as wheel torque commands.

\section{Desired Yaw Moment Calculation}

The wheel torque distribution strategy presented in this paper works while the vehicle is accelerating and cornering. Firstly, a control-oriented vehicle model is built. Based on Figure 3, the yaw motion of the vehicle can be written as:

$$
M=I_{z} \dot{\Omega}
$$

where $M$ is the generalized external moment acting about the $Z$-axis, $I_{z}$ is vehicle moment of inertia about the $Z$-axis, $\Omega$ is the yaw rate of the vehicle.

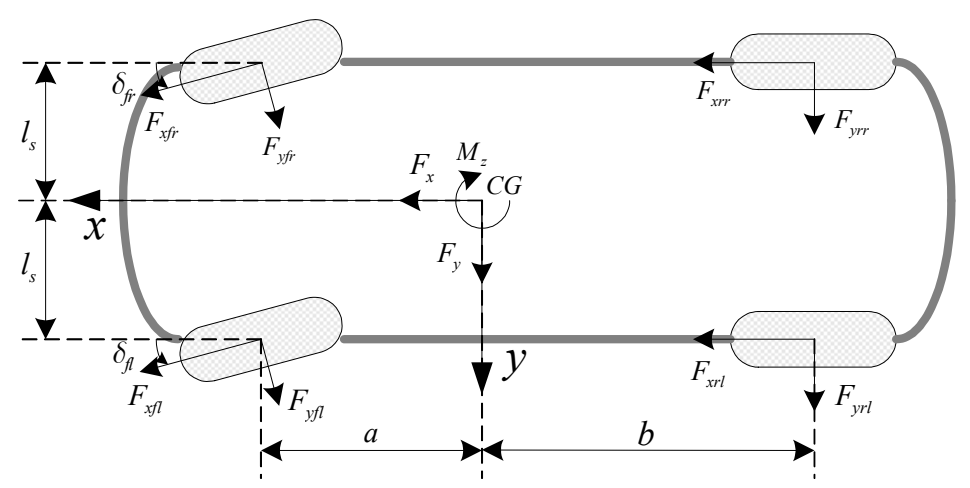

Figure 3. Coordinates for planar motions of the $4 \mathrm{WDEV}$.

On the $4 \mathrm{WDEV}$, the accelerator pedal input and steering wheel input can be used to recognize driver intention. In the high-layer of the control strategy, the desired torque is figured out from driver input. A model suggested in [15] is used as reference model of desired yaw rate as follows:

$$
\frac{\Omega_{d}(\mathrm{~s})}{\Theta_{s w}(\mathrm{~s})}=\frac{\widehat{V}_{x}}{\left(\tau_{r} \mathrm{~s}+1\right)\left(\tau_{d} \mathrm{~s}+1\right)}
$$

where $\widehat{V}_{x}=\frac{k_{r} V_{x}}{\left(1+k_{a} V_{x}^{2}\right) l} G R . \Omega_{d}$ is the desired vehicle yaw rate, $\Theta_{s w}$ is the steering wheel input, $t_{r}$ and $\tau_{d}$ are the time constants, $V_{x}$ is the vehicle velocity along the $X$-axis, $k_{r}$ is the gain of the reference model, $k_{a}$ is the stability factor, $l$ is the vehicle wheelbase, $G R$ is the gear ratio of the front steering mechanism linkage. 
The difference between the desired yaw rate and observed value by sensors is used to calculate the desired yaw moment based on SMC as follows. In the SMC framework adopted in this work, the control objective is to reach and remain in sliding surface $e=0$, where:

$$
e=\Omega-\Omega_{d}+\int\left(\Omega-\Omega_{d}\right) d t
$$

First-order asymptotically stable desired error dynamics is defined as follows:

$$
\dot{e}=-k \operatorname{sgn}(e)
$$

where $k$ is the gain factor.

Then the desired yaw moment $M_{d}$ is:

$$
M_{d}=I_{z}\left[\dot{\Omega}_{d}-\left(\Omega-\Omega_{d}\right)-k \operatorname{sgn}(e)\right]
$$

and:

$$
\begin{aligned}
M= & F_{x f l}\left(\operatorname{asin} \delta_{f l}-l_{s} \cos \delta_{f l}\right)+F_{y f l}\left(a \cos \delta_{f l}+l_{s} \sin \delta_{f l}\right)+F_{x f r}\left(\operatorname{asin} \delta_{f r}+l_{s} \cos \delta_{f r}\right) \\
& +F_{y f r}\left(a \cos \delta_{f r}-l_{s} \sin \delta_{f r}\right)-F_{x r l} l_{s}+F_{y r l} b+F_{x r r} l_{s}-F_{y r r} b
\end{aligned}
$$

where $F_{x}$ is the tire longitudinal force, $F_{y}$ is the tire lateral force. a, b is the distance from vehicle center of gravity (c.g.) to the front and rear axle, respectively. $\delta_{f l}$ and $\delta_{f r}$ are the steering angle of the front left and front right wheel, respectively. The track is shown in Figure 3 as $2 l_{s}$. The corner marks $f l, f r, r l, r r$ mean the front left, front right, rear left and rear right wheel, respectively.

The simplified equation of motion for wheel is:

$$
T_{m i} i_{r}-F_{x i} R_{r}=J_{\omega} \dot{\omega}_{i}
$$

where $T_{m i}$ is the motor torque, $i_{r}$ is the gear ratio, $R_{r}$ is the wheel radius, $J_{\omega}$ is the wheel rotational inertia, $\omega_{i}$ is wheel angular velocity and $i=1,2,3$ or 4 corresponds to the $f l, f r, r l$ or $r r$ wheel, respectively.

When $\lambda \ll 1, J \dot{\omega}_{i}$ is sufficiently small compared to $T_{m i} i_{r}$ and $F_{x i} R_{r}$, therefore:

$$
T_{m i} i_{r} \approx F_{x i} R_{r}
$$

Then we can get:

$$
\begin{aligned}
& T_{m f l}\left(\operatorname{asin} \delta_{f l}-\mathrm{L}_{s} \cos \delta_{f l}\right)+T_{m f r}\left(\mathrm{~L}_{s} \cos \delta_{f r}+\operatorname{asin} \delta_{f r}\right)+T_{m r l}\left(-\mathrm{L}_{s}\right)+T_{m r r} \mathrm{~L}_{s} \\
& \quad=M_{d} R_{r}-F_{y f l} R_{r}\left(\operatorname{acos} \delta_{F}+\mathrm{L}_{s} \sin \delta_{F}\right)-F_{y f r} R_{r}\left(\operatorname{acos} \delta_{R}-\mathrm{L}_{s} \sin \delta_{R}\right)+F_{y r l} b R_{r}+F_{y r r} b R_{r}
\end{aligned}
$$

As the developed controller works when the vehicle is driven in the linear region with small tire slip angle, the following approximation is used here:

$$
F_{y i}=-C_{i} \alpha_{i}
$$

where $C_{i}$ is the wheel cornering stiffness, $\alpha_{i}$ is the tire slip angle. $C_{i}$ and $\alpha_{i}$ can be obtained by a sensor or observer. Thus we can get an equation which defines the ideal relation between wheel driving torque $T_{m i}$ and desired vehicle yaw rate $\Omega_{d}$ : 


$$
\begin{aligned}
\sum_{i=1}^{4} T_{m i} L_{i}= & I_{z} R_{r}\left[\dot{\Omega}_{d}-\left(\Omega-\Omega_{d}\right)-k \operatorname{sgn}(\mathrm{e})\right] \\
& -F_{y f l} R_{r}\left(\operatorname{acos} \delta_{f l}+\mathrm{L}_{s} \sin \delta_{f l}\right)-F_{y f r} R_{r}\left(\operatorname{acos} \delta_{f r}-\mathrm{L}_{s} \sin \delta_{f r}\right)+F_{y r l} b R_{r}+F_{y r r} b R_{r}
\end{aligned}
$$

In addition, we can get the total driving torque $T_{d}$ requested by the driver from:

$$
T_{d}=4 P T_{m \max }
$$

where $P$ is the throttle opening, $T_{m \text { max }}$ is the motor peak torque. From Equations (11) and (12) we can get the constraints for wheel driving torque. It is important to note here that Equation (11) does not have to be met.

\section{Wheel Torque Control Allocation}

\subsection{The Multi-Objective Optimization Algorithm}

In the low-layer of the proposed wheel torque control strategy, a multi-objective mathematical programming method is adopted. The penalty function consisting of yaw moment control offset, drive system energy loss and slip ratio constraint is shown as follows:

$$
\begin{aligned}
& \min _{T_{m i}} J=\left(\sum_{i=1}^{4} T_{m i} L_{i}-V_{d}\right)^{2}+\sigma_{p} \sum_{i=1}^{4} \mathrm{C}_{p}\left(T_{m i}\right)+\sigma_{t} \mathrm{C}_{t}\left(T_{m i}\right) \\
& \text { s.t. } \sum_{i=1}^{4} T_{m i}=T_{d} ; \\
& \quad T_{m i \min } \leq T_{m i} \leq T_{m i \max }, i=1,2,3,4 .
\end{aligned}
$$

The first part of the penalty function $J$ is yaw moment control offset:

$$
\Delta_{M}=\left(\sum_{i=1}^{4} T_{m i} L_{i}-V_{d}\right)^{2}
$$

where: $\mathrm{L}_{1}=\operatorname{asin} \delta_{F}-\mathrm{L}_{s} \cos \delta_{F}, \mathrm{~L}_{2}=\mathrm{L}_{s} \cos \delta_{R}+\operatorname{asin} \delta_{R}, \mathrm{~L}_{3}=-\mathrm{L}_{s}, \mathrm{~L}_{4}=\mathrm{L}_{s}$, $V_{d}=M_{d} R_{r}-F_{y 1} R_{r}\left(\operatorname{acos} \delta_{F}+\mathrm{L}_{s} \sin \delta_{F}\right)-F_{y 2} R_{r}\left(\operatorname{acos} \delta_{R}-\mathrm{L}_{s} \sin \delta_{R}\right)+F_{y 3} b R_{r}+F_{y 4} b R_{r}$.

This is the difference between the yaw moment generated by the current torque distribution point and the desired yaw moment from high-layer yaw motion controller. This is used to control the vehicle yaw rate, which will optimize the vehicle cornering response.

The second part is the energy loss of the drive system at the current torque distribution point, which consists of copper loss, iron loss, inverter loss, friction loss, stray loss and transmission loss:

$$
\mathrm{C}_{p}\left(T_{m i}\right)=\mathrm{a}_{3} T_{m i}{ }^{3}+\mathrm{a}_{2} T_{m i}{ }^{2}+\mathrm{a}_{1} T_{m i}+\mathrm{a}_{0}
$$

where $a_{3}, a_{2}, a_{1}$ and $a_{0}$ are the fitting coefficients, they are fitted out with the experimental data shown in Figure 4. 


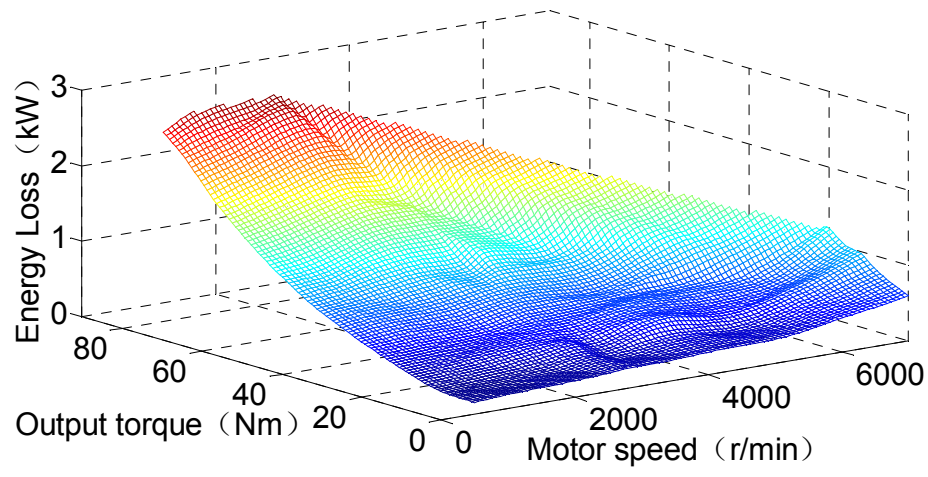

Figure 4. Energy loss vs. motor speed and output torque.

In this paper the expression Equation (15) is used to perform the energy-saving control in the penalty function and it is different from that used in [11-14] shown as below:

$$
\mathrm{C}_{p}\left(T_{m i}\right)=9549 \sum_{i=1}^{4} \frac{T_{m i} n_{i}}{\eta\left(T_{m i}\right)}
$$

In the above Equation (16), $n_{i}$ is the motor speed and $\eta\left(T_{m i}\right)$ is a function with relatively high order that will lead to high calculation cost when solving the constructed mathematical programming. Therefore, the expression Equation (15) with relatively low order is used as a part of the penalty function.

The third part of the penalty function is used to put constraint on the wheel slip ratio:

$$
\mathrm{C}_{t}\left(T_{m i}\right)=\sum_{i=1}^{4}\left(T_{m i} \lambda_{i}\right)^{2}
$$

where $\lambda_{i}$ is the wheel slip ratio. This item is used for avoiding excessive spin of wheels by reducing the driving torque on the wheels that have relatively high slip ratios.

\subsection{Off-Line and On-Line Optimization}

As the penalty function in Equation (13) is non-convex and nonlinear, the algorithms used in the literature to search for the global optimal point will lead to heavy calculation burdens and are thus not suitable for practical application. In this paper, the combination of off-line and on-line optimization is used to search for the optimal point of Equation (13), where, off-line optimization based on a simplified penalty function is used to find the optimal point considering yaw moment and energy-saving control, the following on-line optimization is to search for the local optimal point near the off-line result with consideration of the slip ratio constraint. The multi-objective programming is simplified as below, and it is suitable for off-line optimization:

$$
\begin{aligned}
& \min _{T_{m i}} J_{1}=\mathrm{C}_{p}\left(T_{m 1}\right)+\mathrm{C}_{p}\left(T_{m 3}\right), \quad \min _{T_{m i}} J_{2}=\mathrm{C}_{p}\left(T_{m 2}\right)+\mathrm{C}_{p}\left(T_{m 4}\right) \\
& \text { s.t. } T_{m 1}+T_{m 3}=\left(T_{d}-M_{d} / \mathrm{L}_{s}\right) / 2 ; \\
& T_{m 2}+T_{m 4}=\left(T_{d}+M_{d} / \mathrm{L}_{s}\right) / 2 ; \\
& \quad T_{m i \min } \leq T_{m i} \leq T_{m i \max }, i=1,2,3,4 .
\end{aligned}
$$

During the simplification, the steering angle is approximated as zero and the slip ratio constraint is neglected. At a certain motor speed the energy loss function $\mathrm{C}_{p}\left(T_{m i}\right)$ is confirmed, then the problem 
Equation (18) can easily be solved by off-line optimization. It is important to note here that in [16], the authors had done some research on the distribution of driving torque between the front axle and rear axle of a four-wheel-drive electric vehicle. In the development of the energy saving-oriented wheel torque distribution controllers, the functional relationship between energy efficiency and drive train output torque is needed. The accuracy of the distribution results must rely on that of the function established. In [16], a second-order function was deduced to describe the relationship between energy loss and drive train output torque based on theoretical analysis. Then the authors drew the conclusion that wheel torque equal division had better effect in vehicle energy saving than control allocation algorithms introduced in the literature. In this paper, the energy efficiency of an electric drive system was gotten based on a dynamometer system, and the functions of energy loss versus output torque are fitted out by the experimental data. In Figure 5, the accuracy of second-order function is compared with the third-order one at different motor speeds.

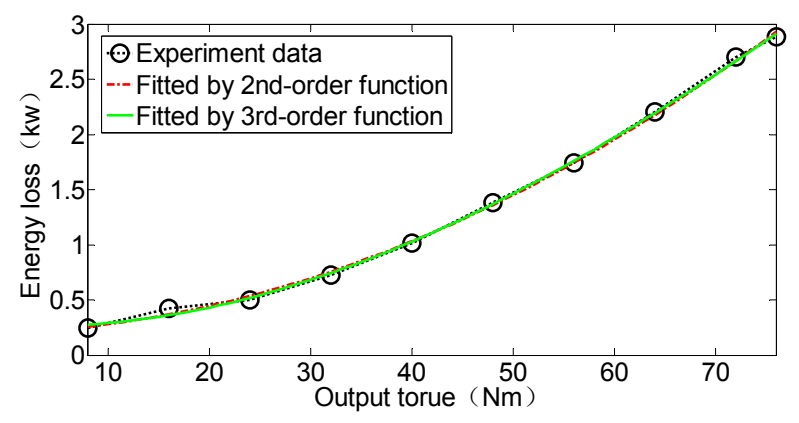

(a) The motor speed is $2,000 \mathrm{rpm}$.

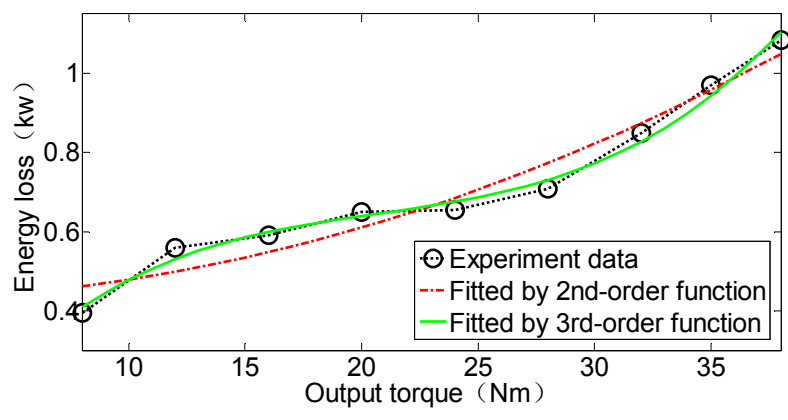

(b) The motor speed is 3,000 rpm.

Figure 5. The fitting function of energy loss vs. output torque.

It can be seen from Figure 5 that at 2,000 rpm, both the second-order and third-order functions have high accuracy in the description of the relationship between drive system energy loss and its output torque. At 3,000 rpm, the third-order function is much more accurate than the second-order one. Therefore, in this paper the function adopted in describing the relationship between energy loss and output torque is third-order when the motor speed is $3,000 \mathrm{rpm}$ or above.

Through off-line optimization the partition factor between $T_{m 1}$ and $T_{m 3}$ or $T_{m 2}$ and $T_{m} 4$ can be obtained at different motor speed $\times$ and torque demand $\times$, which is shown in Figure 6 . We can know that under most conditions including low speed and low desired driving torque conditions, the partition factor between two motors is 0.5 . When the motor speed ranges from 3,500 rpm to 7,000 rpm and the desired torque for the two motors from either side of the vehicle is relatively small, the partition factor is 0 , which means in this area the desired driving torque should be generated by one of the two motors. 


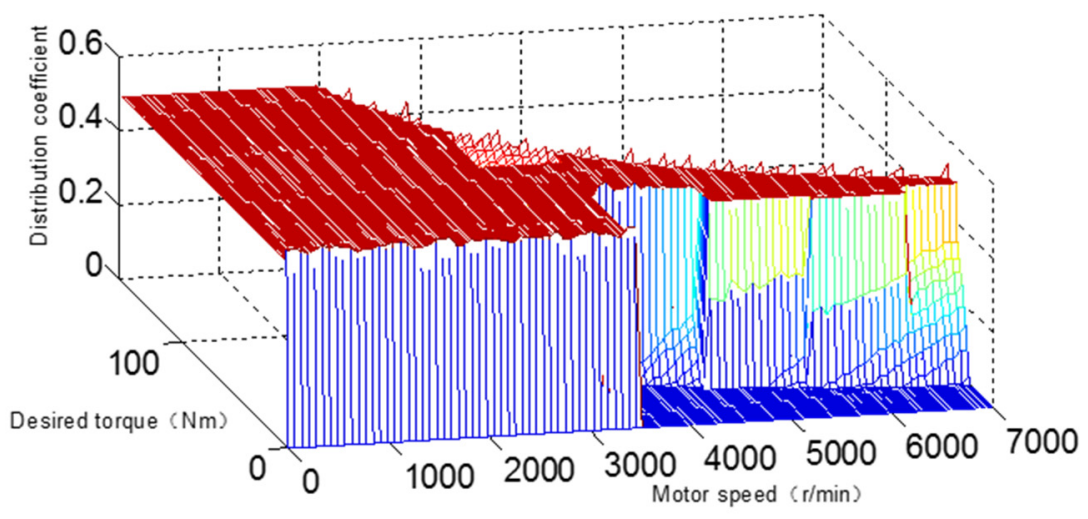

Figure 6. The partition factor based on off-line calculation.

The off-line optimization result above, named $T_{m}{ }^{*}$, will be used as the starting point in the on-line optimization by a 2-D lookup table. One of the inputs of the lookup table is the summation driving torque $T_{\text {suml }}$ or $T_{\text {sumr }}$ of the two motors in the left or right side of the vehicle, and the other one is the vehicle velocity from which we can get the motor speed. The partial factor shown in Figure 6 is used for the 2-D lookup table. The mentioned summation driving torque $T_{\text {suml }}$ and $T_{\text {sumr }}$ is figured out by the following expressions:

$$
T_{\text {suml }}=T_{m 1}+T_{m 3}=\left(T_{d}-M_{d} / \mathrm{L}_{s}\right) / 2, T_{\text {sumr }}=T_{m 2}+T_{m 4}=\left(T_{d}+M_{d} / \mathrm{L}_{s}\right) / 2
$$

In order to take the steering wheel angle and slip ratio constraint into consideration in wheel torque distribution, an on-line optimization step is added to the control allocation process. Newton-Lagrange [17] is used to find the local optimal point around $T_{m}{ }^{*}$. The adopted algorithm can transform the nonlinear and non-convex optimization to quadratic programming at $T_{m}{ }^{*}$ shown as below. First, the problem is simplified by removing the numerical limit on wheel torque:

$$
\min J\left(T_{m}\right)=\left(\sum_{i=1}^{4} T_{m i} L_{i}-V_{d}\right)^{2}+\sigma_{p} \sum_{i=1}^{4} \mathrm{C}_{p}\left(T_{m i}\right)+\sigma_{t} \mathrm{C}_{t}\left(T_{m i}\right), \text { s.t. } \sum_{i=1}^{4} T_{m i}=T_{d} .
$$

Then the Lagrange function can be written as:

$$
L\left(T_{m}, \mu\right)=J\left(T_{m}\right)+\mu h\left(T_{m}\right)
$$

where:

$$
h\left(T_{m}\right)=\sum_{i=1}^{4} T_{m i}-T_{d}
$$

Thus, the local optimal point around $T_{m}{ }^{*}$ can be figured out by the equation as follows:

$$
\left[\begin{array}{cc}
W\left(T_{m}, \mu\right) & -\nabla h\left(T_{m}\right) \\
-\nabla h\left(T_{m}\right) & 0
\end{array}\right]\left[\begin{array}{l}
d_{k} \\
\nu_{k}
\end{array}\right]=\left[\begin{array}{c}
-\nabla J\left(T_{m}\right)+\nabla h\left(T_{m}\right) \mu_{k} \\
h\left(T_{m}\right)
\end{array}\right]
$$

where, $W\left(T_{m}, \mu\right)$ is the Hessian matrix of the Lagrange function with respect to $T_{m} . d_{k}$ and $\nu_{k}$ are the variation of $T_{m}$ and $\mu$, respectively:

$$
W\left(T_{m}, \mu\right)=\nabla_{T T}^{2} L\left(T_{m}, \mu\right)=\nabla_{T T}^{2} J\left(T_{m}\right)+\mu \nabla_{T T}^{2} h\left(T_{m}\right)
$$


From Equation (21) we can get:

$$
\nabla_{T T}^{2} h\left(T_{m}\right)=0
$$

thus:

$$
\begin{gathered}
W\left(T_{m}, \mu\right)=\nabla_{T T}^{2} J\left(T_{m}\right) \\
\nabla J\left(T_{m}\right)=\left[\begin{array}{llll}
\nabla_{1} J\left(T_{m}\right) & \nabla_{2} J\left(T_{m}\right) & \nabla_{3} J\left(T_{m}\right) & \nabla_{4} J\left(T_{m}\right)
\end{array}\right]^{T} \\
\nabla_{T T}^{2} J\left(T_{m}\right)=\left[\begin{array}{llll}
\nabla_{11}^{2} J\left(T_{m}\right) & \nabla_{12}^{2} J\left(T_{m}\right) & \nabla_{13}^{2} J\left(T_{m}\right) & \nabla_{14}^{2} J\left(T_{m}\right) \\
& \nabla_{22}^{2} J\left(T_{m}\right) & \nabla_{23}^{2} J\left(T_{m}\right) & \nabla_{24}^{2} J\left(T_{m}\right) \\
& & \nabla_{33}^{2} J\left(T_{m}\right) & \nabla_{34}^{2} J\left(T_{m}\right) \\
& & & \nabla_{44}^{2} J\left(T_{m}\right)
\end{array}\right]
\end{gathered}
$$

where:

$$
\begin{gathered}
\nabla_{i i}^{2} J\left(T_{m}\right)=2 \mathrm{~L}_{i}^{2}+\sigma_{p} \nabla_{i i}^{2} \mathrm{C}_{p}\left(T_{m}\right)+2 \sigma_{t} \lambda_{i}^{2} \\
\nabla_{i j}^{2} J\left(T_{m}\right)=2 \mathrm{~L}_{i} \mathrm{~L}_{j}
\end{gathered}
$$

therefore:

$$
\boldsymbol{\Delta}_{\mathbf{T}}^{\mathrm{T}} W\left(T_{m}, \mu\right) \boldsymbol{\Delta}_{\mathbf{T}}=2\left(\sum_{i=1}^{4} \Delta_{T_{i}} \mathrm{~L}_{i}\right)^{2}+\sum_{i=1}^{4}\left(\sigma_{p} \nabla_{i i}^{2} \mathrm{C}_{p}\left(T_{m}\right)+2 \sigma_{t} \lambda_{i}^{2}\right) \Delta_{T_{i}}^{2}
$$

We can see from (30), $\forall \boldsymbol{\Delta}_{\mathbf{T}} \neq 0, \boldsymbol{\Delta}_{\mathbf{T}}{ }^{\mathrm{T}} W\left(T_{m}, \mu\right) \boldsymbol{\Delta}_{\mathbf{T}}>0$ can be ensured by properly choosing $\sigma_{p}$ and $\sigma_{t}$, so $W\left(T_{m}, \mu\right)$ is a positive definite matrix. Therefore, we can get the solution of Equation (22) by solving the convex optimization problem below:

$$
\left\{\begin{aligned}
\min q_{k}(\Delta)= & \frac{1}{2} \Delta^{\mathrm{T}} W\left(T_{m}{ }^{*}, \mu^{*}\right) \Delta+\nabla J\left(T_{m}{ }^{*}\right)^{\mathrm{T}} \Delta \\
\text { s.t. } & h\left(T_{m}^{*}\right)+\nabla h\left(T_{m}^{*}\right)^{\mathrm{T}} \Delta=0 .
\end{aligned}\right.
$$

In this paper, the fixed-point algorithm in $[18,19]$ is adopted to solve the problem above. $\Delta$ can be used as optimal direction of $T_{m}{ }^{*}$. The optimum step $\alpha$ is figured out by setting a penalty function as follows:

$$
\begin{gathered}
\mathrm{P}\left(T_{m}, \mu\right)=\left\|\nabla \mathrm{L}\left(T_{m}, \mu\right)\right\|^{2}=\left\|\nabla \mathrm{J}\left(T_{m}\right)+\nabla \mathrm{h}\left(T_{m}\right) \mu\right\|^{2}+\left\|\mathrm{h}\left(T_{m}\right)\right\|^{2} \\
\alpha=\rho^{m} \Delta
\end{gathered}
$$

where, $0<\rho<1, m$ is the smallest non-negative integer that satisfies the following equations:

$$
\left\{\begin{array}{c}
\mathrm{P}\left(T+\rho^{m} d, \mu+\rho^{m} \nu\right) \leq\left(1-\gamma \rho^{m}\right) \mathrm{P}(T, \mu) \\
T_{\min } \leq T+\rho^{m} d \leq T_{\max }
\end{array}\right.
$$

Then the result of on-line optimization is $T_{m}{ }^{*}+\alpha$. The off-line optimization has given out the wheel torque control allocation result considering yaw moment control and energy saving, but without 
considering the steering angle and slip ratio constraint. In the on-line optimization, the actual steering angle is considered, as well as the slip ratio constraint, which has made the penalty function more complicated. Newton-Lagrange can transform such a nonlinear and non-convex optimization to a convex one at the off-line optimization result $T_{m}{ }^{*}$, which will bring down the calculation cost.

\section{Simulation and Discussion}

Co-simulation based on MATLAB ${ }^{\circledR}$ and Carsim $^{\circledR}$ is carried out to verify the proposed control strategy. The parameters of the target car are shown in Table 1.

Table 1. Vehicle Parameters.

\begin{tabular}{cccc}
\hline Parameters & Values & Parameters & Values \\
\hline Vehicle Mass & $1486 \mathrm{~kg}$ & Motor Number & 4 \\
Wheelbase & $2.578 \mathrm{~m}$ & Motor Rated Power & $8 \mathrm{~kW}$ \\
Vehicle Moment of Inertia & $2023 \mathrm{~kg} \cdot \mathrm{m}^{2}$ & Motor Peak Torque & $78 \mathrm{Nm}$ \\
Wheel Rolling Radius & $0.298 \mathrm{~m}$ & Motor Rated Speed & $2900 \mathrm{rpm}$ \\
\hline
\end{tabular}

The vehicle performance, provided by equal division of driving torque, off-line optimization based distribution and combination of off-line and on-line optimization control strategy is simulated under two different conditions. Under the simulated driving conditions, the road friction coefficient is 0.85 , and the steering wheel angle changes as shown in Figure 7. The acceleration pedal in puts for the two conditions are shown in Figure 8a and Figure 9a.

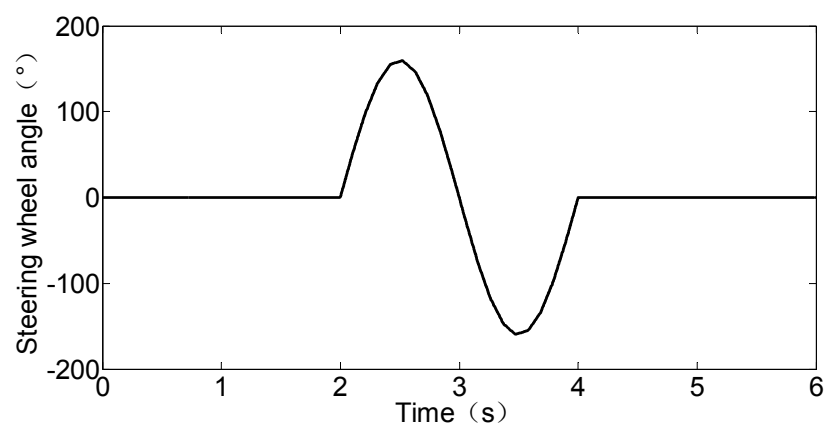

Figure 7. The input of steering wheel during the simulation

\subsection{Large Acceleration Simulation}

Under this condition, the vehicle initial velocity is set to $20 \mathrm{~km} / \mathrm{h}$. 


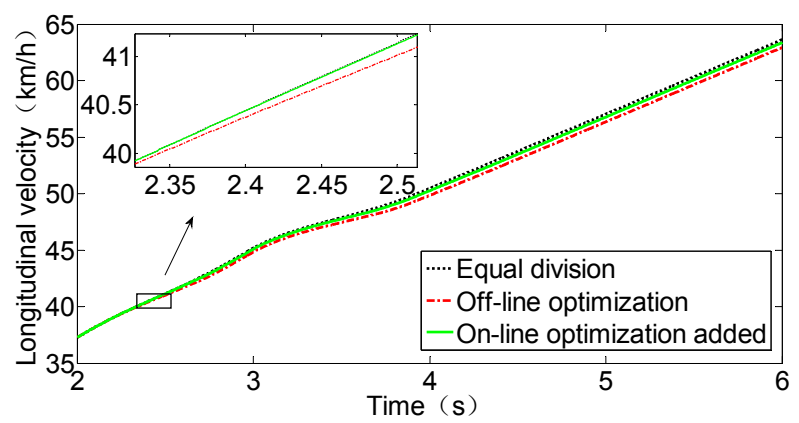

(a) Throttle opening.

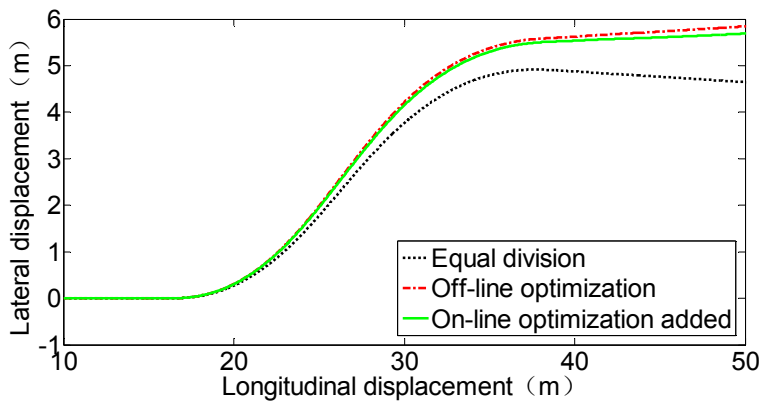

(c) Displacement of the vehicle c.g.

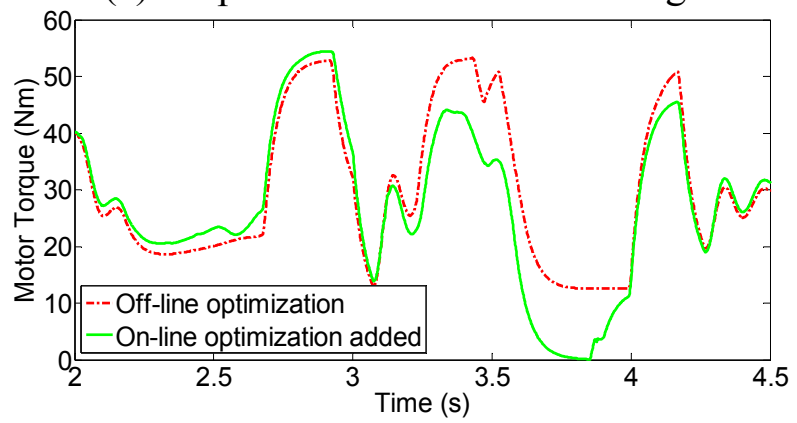

(e) $f l$ wheel driving torque.

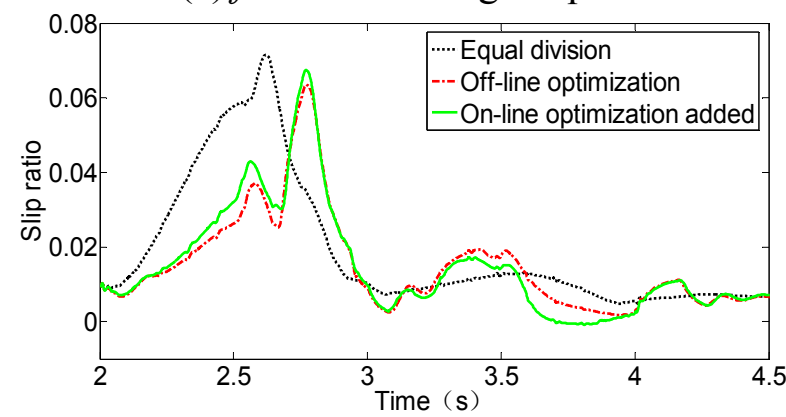

(g) $f l$ wheel slip ratio.

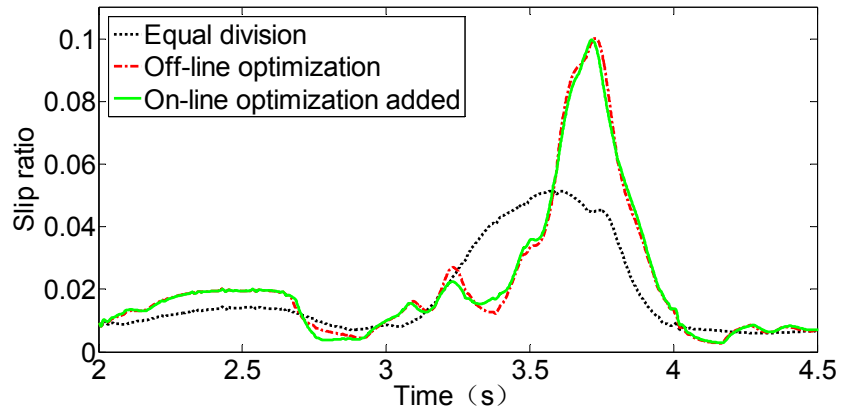

(i) $f r$ wheel slip ratio.

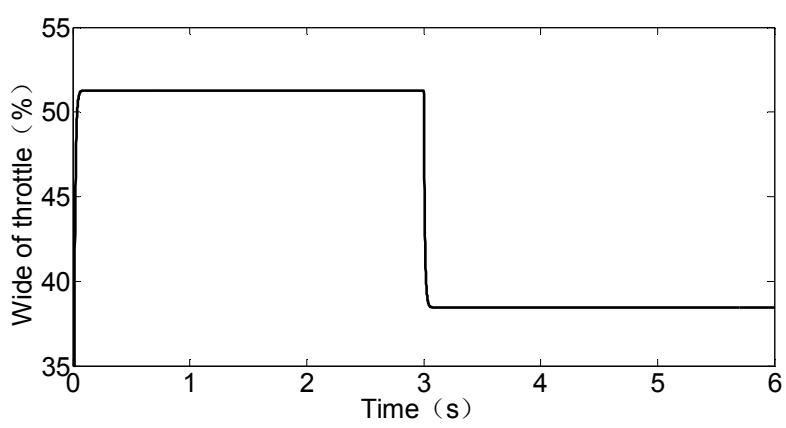

(b) Vehicle longitudinal velocity.

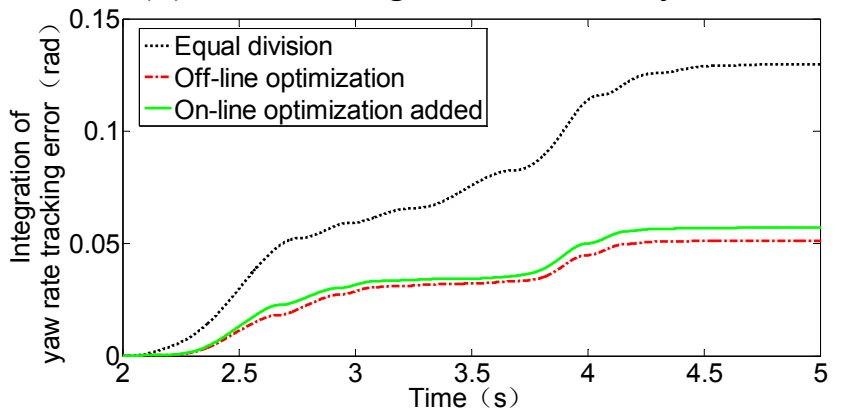

(d) Integration of yaw rate tracking error.

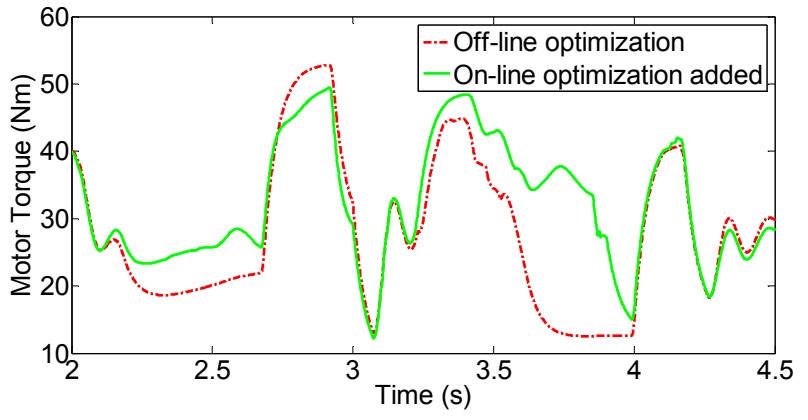

(f) $r l$ wheel driving torque.

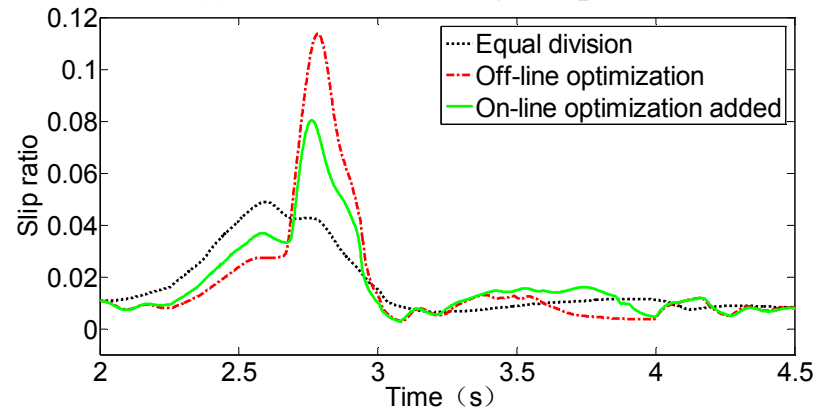

(h) $r l$ wheel slip ratio.

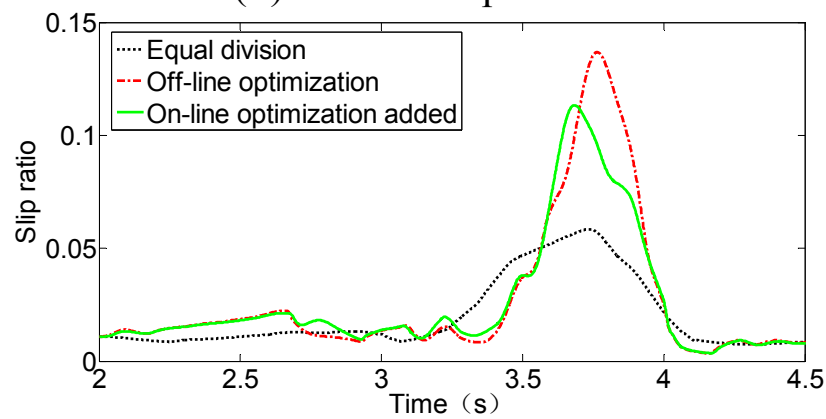

(j) $r r$ wheel slip ratio.

Figure 8. Cont. 


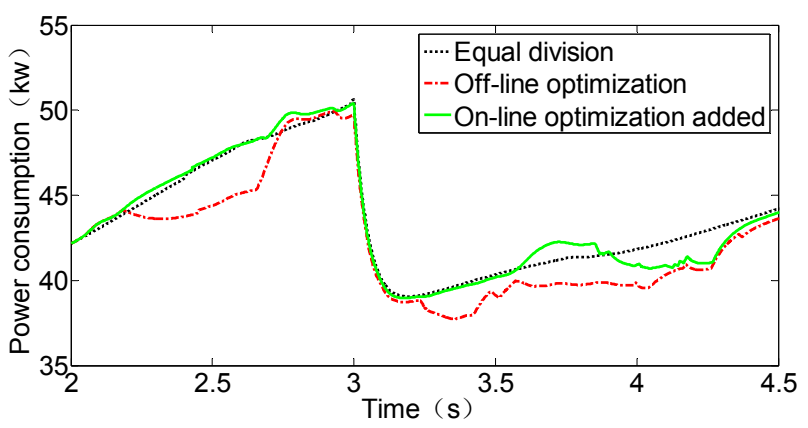

(k) Drive system energy consumption.

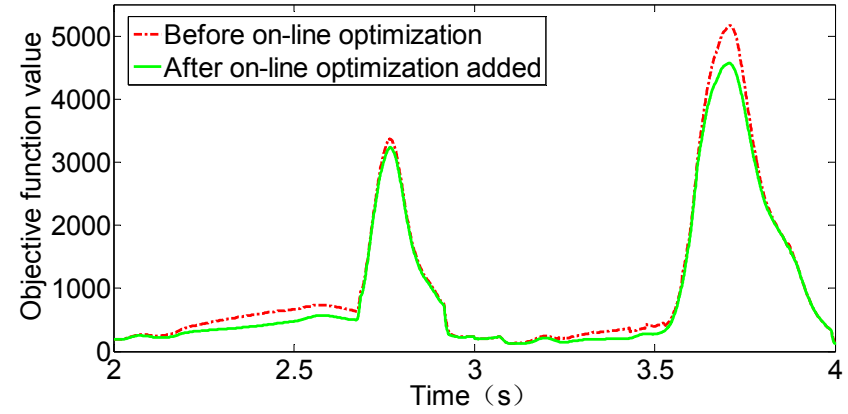

(I) Penalty function value.

Figure 8. The vehicle state under large acceleration.

\subsection{Small Acceleration Simulation}

Under this condition, the vehicle initial velocity is set to $55 \mathrm{~km} / \mathrm{h}$.

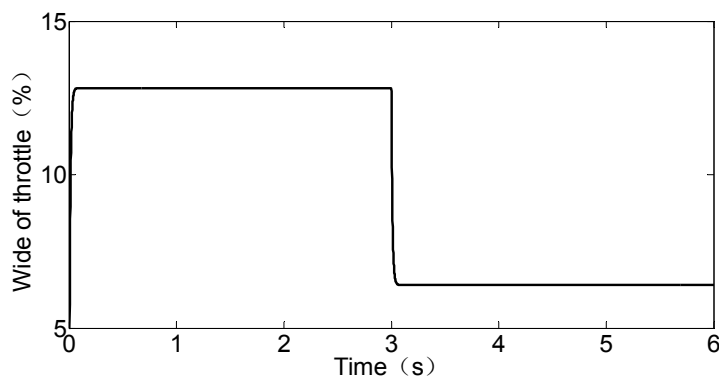

(a) Throttle opening.

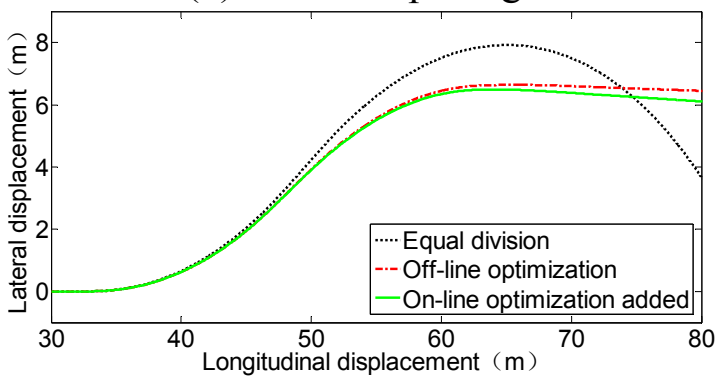

(c) Displacement of vehicle c.g.

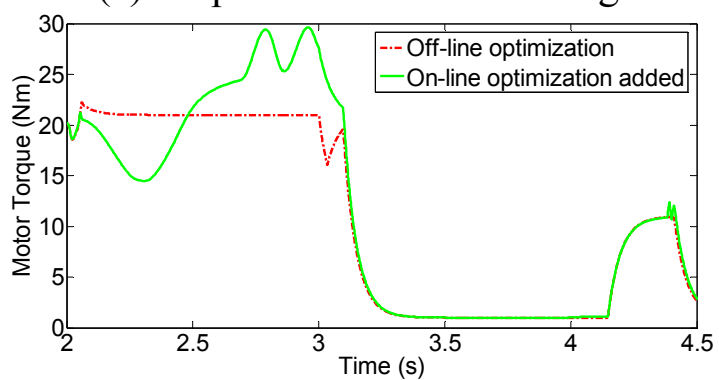

(e) $f l$ wheel driving torque.

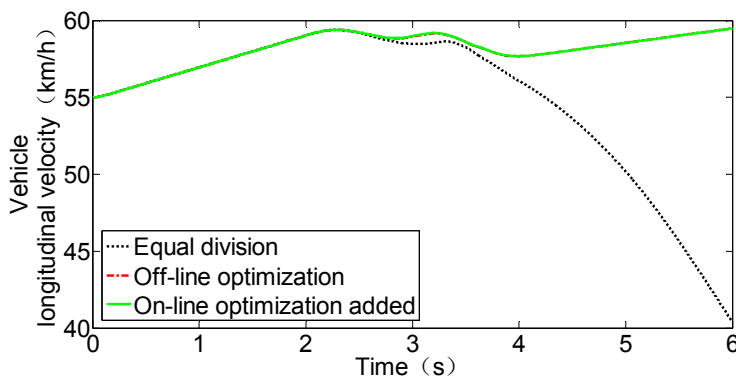

(b) Vehicle longitudinal velocity.

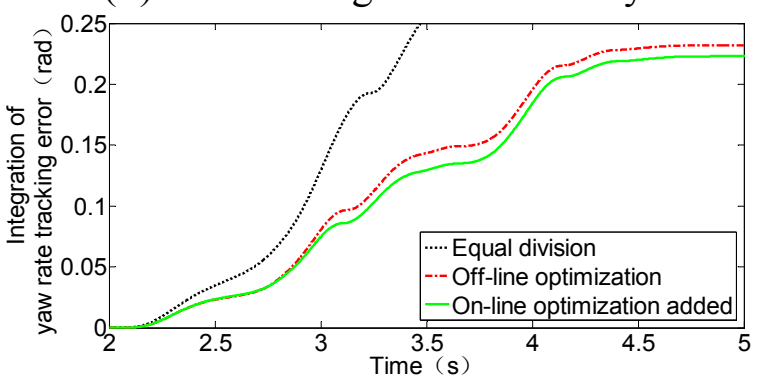

(d) Integration of yaw rate tracking error.

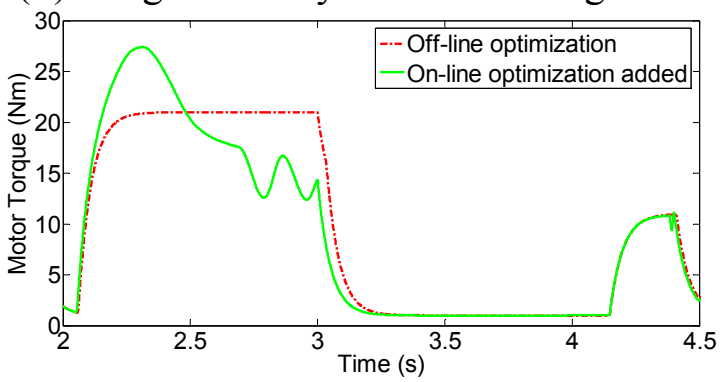

(f) $r l$ wheel driving torque.

Figure 9. Cont. 


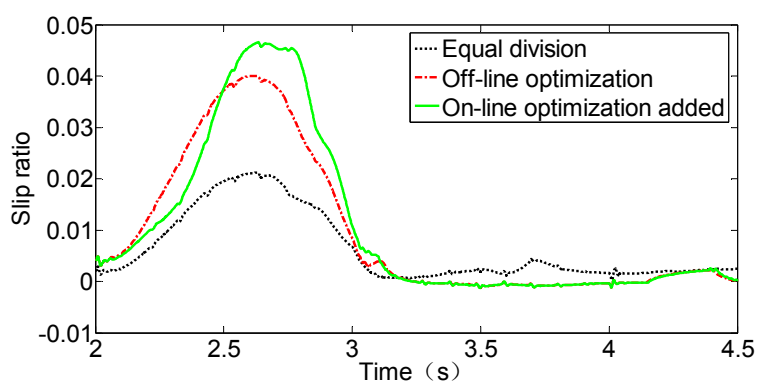

(g) $f l$ wheel slip ratio.

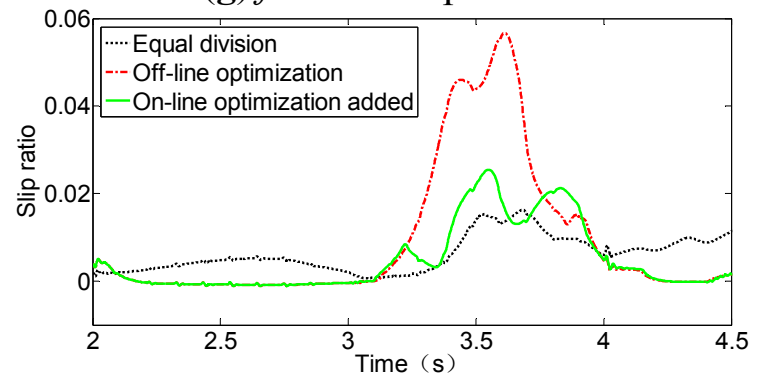

(i) $f r$ wheel slip ratio.

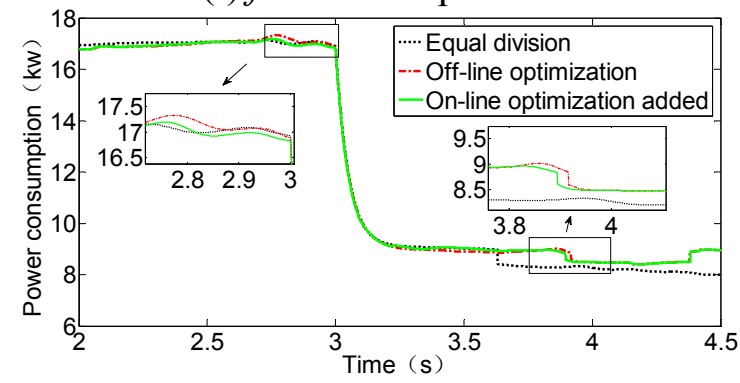

(k) Drive system energy consumption.

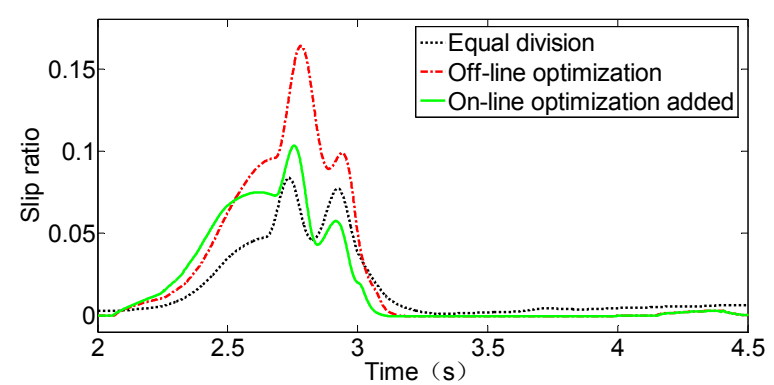

(h) $r l$ wheel slip ratio.

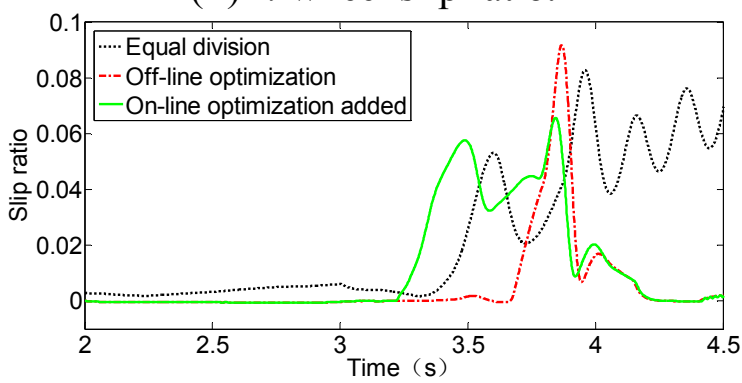

(j) $r r$ wheel slip ratio.

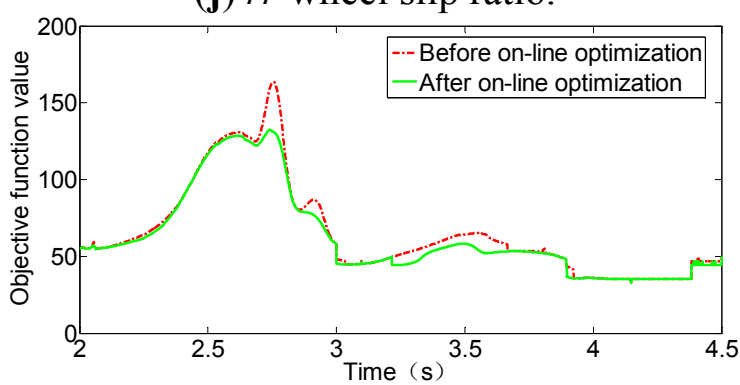

(I) Penalty function value.

Figure 9. The vehicle state under small acceleration.

\subsection{Discussion}

Under the combined condition of accelerating and cornering, the wheel torque control strategy should not only meet the demand of vehicle steering maneuverability improvement and energy savings, but also balance the slip ratio of the four wheels to prevent excessive wheel spin. We can know the vehicle is well controlled under the three control strategies from Figure $8 \mathrm{~b}$ the vehicle velocity time history and $8 \mathrm{c}$ the trajectory of the vehicle c.g.. Figure $8 \mathrm{~d}$ is the integration of yaw rate tracking error, which shows that compared with equal division of wheel torque, either off-line optimization or the combination of off-line and on-line optimization has improved the vehicle tracking ability. This is due to the differential driving performed by the electric drive system when yaw moment is generated. Figure $8 \mathrm{e}, \mathrm{f}$ are the time history of the $f l$ and $r l$ wheel driving torque, respectively, from which we can see how wheel torque changes before and after the introduction of on-line optimization. We can see from Figure $8 \mathrm{~g}-\mathrm{j}$ that the slip ratio of rear wheels is reduced after the on-line optimization. Slip ratio constraint added in the penalty function has balanced the slip level among different wheels. Figure 8k is the energy consumption of the drive system under the three different control strategies, from which we can see that when the driving torque is evenly distributed among the four wheels, the energy consumption is the highest, and off-line optimization-based distribution is the most efficient way for saving energy. It is important to note here 
that, from Figure 6 we know when the motor speed is lower than 3,500 rpm, or car velocity is lower than $53 \mathrm{~km} / \mathrm{h}$, wheel even torque distribution is the most efficient method. In Figure 8k, during the first few seconds before the car accelerates to $53 \mathrm{~km} / \mathrm{h}$, the power consumption of off-line optimization and evenly distributing control are different, though the wheel driving torque on the left or right side is evenly distributed with off-line optimization. This is because the sum of output torque of off-line optimization is lower than that the driver desires. Figure $8 \mathrm{~b}$ tells us that the off-line optimization has led to a lower vehicle velocity than evenly distributing control. Off-line optimization generates the biased torque demand between the left and right vehicle side, and it may result in a big torque command that is beyond the motor capability. Therefore, using off-line optimization can reduce energy consumption and the yaw rate tracking error, but deteriorate the vehicle acceleration performance. Figure 81 shows the change of penalty function that has given consideration to the three targets of vehicle maneuverability improvement, energy consumption reduction and slip ratio balance, before and after the on-line optimization.

When the vehicle velocity is high during cornering, a large yaw moment is needed to generate the desired vehicle yaw rate. The longitudinal force of vehicle will lead to reduction of the lateral force that is important for vehicle cornering. Therefore, yaw moment control based on differential driving is especially important when the vehicle is accelerating while cornering with high velocity. From Figure $9 \mathrm{~b}-\mathrm{d}$ we can find that the vehicle has been in a kind of loss control state when wheel driving torque is evenly distributed. Both the off-line optimization and the proposed strategy have successfully controlled the vehicle in the appropriate state. Figure $9 \mathrm{e}-\mathrm{j}$ also tell us that the on-line optimization has balanced the wheel slip ratios to prevent the excessive spin by reducing wheel driving torque when slip ratio is high. It can be seen from Figure 9k that the energy consumption is diminished when the vehicle is controlled with the proposed strategy, which means the combination of off-line and on-line optimization is better than individual off-line optimization. Figure 91 shows the decrease of the penalty function value after the on-line optimization.

\section{Conclusions}

The research and application of electric drive technology is an effective way to solve energy and environment issues. Moreover, on 4WDEVs the "fun-to-drive" qualities [20] can be realized by properly distributing wheel torque which may also extend the driving range.

In this paper, a vehicle drive control strategy is developed to allow optimal wheel torque distribution under combined conditions while accelerating and cornering. The proposed approach is developed based on a hierarchical structure. At the high-level the desired driving torque and yaw moment are figured out based on SMC due to the model inaccuracy and parameter error. At the low-level the total driving torque is allocated to wheels by multi-objective optimization. The slip ratio constraint has been added to the penalty function to prevent excessive spin. Another two parts of the penalty function are yaw moment control offset and energy loss instead of energy consumption applied in the literature. In this way the penalty function is simplified which makes the calculation cost reduced. The multi-objective programming is solved based on a combination of off-line optimization and on-line optimization algorithm. At the first process, off-line optimization is carried out based on the simplified penalty function, and the result has been used as the start point of on-line optimization. During the on-line optimization, local minimal point is reached around the off-line result. Co-simulation based on 
MATLAB $^{\circledR}$ and Carsim ${ }^{\circledR}$ has verified the presented wheel torque distribution process in terms of both maneuverability control and energy savings. The verification based on vehicle test will be carried out after a $4 \mathrm{WDEV}$ is developed.

\section{Acknowledgments}

This work was supported by National Natural Science Foundation of China (no.51175043).

\section{Author Contributions}

Lin Cheng and Zhifeng $\mathrm{Xu}$ built the dynamic model of vehicle dynamics control for 4WDEV, developed the wheel torque control strategy and performed the simulations.

\section{Conflicts of Interest}

The authors declare no conflict of interest.

\section{References}

1. Wu, T.; Zhang, M.B.; Ou, X.M. Analysis of future vehicle energy demand in China based on a Gompertz function method and computable general equilibrium model. Energies 2014, 7, 7454-7482.

2. He, H.W.; Peng, J.K.; Xiong, R.; Fan, H. An acceleration slip regulation strategy for four-wheel drive electric vehicles based on sliding mode control. Energies 2014, 7, 3748-3763.

3. Sakai, S.; Sado, H.; Hori, Y. Motion control in an electric vehicle with four independently driven in-wheel motors. IEEE/ASME Trans. Mechatron. 1999, 4, 9-16.

4. Lin, C.; Zhang, Z.J.; Ma, J. Dual-motor Anti-slip Differential Drive System. Chinese Patent China 200810097693.5, 2009.

5. Jeongmin, K.; Chiman, P. Control algorithm for an independent motor-drive vehicle. IEEE Trans. Veh. Technol. 2010, 59, 3213-3222.

6. Li, F.Q.; Wang, J.; Liu, Z.D. Motor torque based vehicle stability control for four-wheel-drive electric vehicle. In Proceedings of the IEEE Vehicle Power and Propulsion Conference, Dearborn, MI, USA, 7-11 September 2009; pp. 1596-1601.

7. Peng, H.; Hori, Y. Optimum traction force distribution for stability improvement of $4 \mathrm{WDEV}$ in critical driving condition. In Proceedings of the 9th IEEE International Workshop on Advanced Motion Control, Istanbul, Turkey, 27-29 March 2006; pp. 596-601.

8. He, Z.Y.; Ji, X.W. Nonlinear robust control of integrated vehicle dynamics. Veh. Syst. Dyn. 2012, 50, 247-280.

9. Falcone, P.; Tseng, H.E.; Borrelli, F.; Asgari, J.; Hrovat, D. MPC-based yaw and lateral stabilization via active front steering and braking. Veh. Syst. Dyn. 2008, 46, 611-628.

10. Javad, A.; Ali, K.S.; Mansour, K. Adaptive vehicle lateral-plane motion control using optimal tire friction forces with saturation limits consideration. IEEE Trans. Veh. Technol. 2009, 58, 4098-4107. 
11. Wang, R.R.; Chen, Y.; Feng, D.W. Development and performance characterization of an electric ground vehicle with independently actuated in-wheel motors. J. Power Sources 2011, 196, 3962-3971.

12. Chen, Y.; Wang, J.M. Energy-efficient control allocation with applications on planar motion control of electric ground vehicles. In Proceeding of the 2011 American Control Conference, San Francisco, CA, USA, 29 June-1 July 2011; pp. 2719-2724.

13. Chen, Y.; Wang, J.M. Design and experimental evaluations on energy efficient control allocation methods for over actuated electric vehicles: Longitudinal motion case. IEEE/ASME Trans. Mechatron. 2014, 19, 538-548.

14. Leonardo, D.N.; Aldo, S.; Patrick, G. Wheel torque distribution criteria for electric vehicles with torque-vectoring differentials. IEEE Trans. Veh. Technol. 2014, 63, 1593-1602.

15. Horiuchi, S.; Okada, K.; Nohtomi, S. Improvement of vehicle handling by nonlinear integrated control of four wheel steering and four wheel torque. JSAE Rev. 1999, 20, 459-464.

16. Gu, J.; Ouyang, M.; Lu, D. Energy efficiency optimization of electric vehicle driven by in-wheel motor. Int. J. Automot. Technol. 2013, 14, 763-772.

17. Huang, H.X.; Han, J.Y. Mathematical Programming; Tsinghua University Press: Beijing, China, 2006; pp. 289-291.

18. Lu, P. Constrained tracking control of nonlinear systems. Syst. Control Lett. 1997, 27, 305-314.

19. Wang, J.M. Coordinated and Reconfigurable Vehicle Dynamics Control; The University of Texas at Austin: Austin, TX, USA, 2007; pp. 80-104.

20. Novellis, L.D.; Sorniotti, A.; Gruber, P. Optimal wheel torque distribution for a four-wheel-drive fully electric vehicle. SAE Int. J. Passeng. Cars-Mech. Syst. 2013, 6, 128-136.

(C) 2015 by the authors; licensee MDPI, Basel, Switzerland. This article is an open access article distributed under the terms and conditions of the Creative Commons Attribution license (http://creativecommons.org/licenses/by/4.0/). 